\title{
Evolution of gremlin 2 in cetartiodactyl mammals: gene loss coincides with lack of upper jaw incisors in ruminant
}

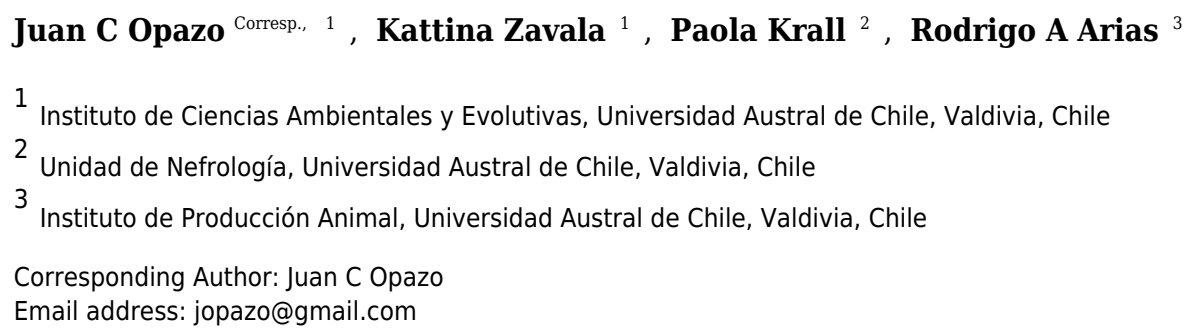

Understanding the processes that give rise to genomic variability in extant species is an active area of research within evolutionary biology. With the availability of whole genome sequences, it is possible to quantify different forms of variability such as variation in gene copy number, which has been described as an important source of genetic variability and in consequence of phenotypic variability. Most of the research on this topic has been focused on understanding the biological significance of gene duplication, and less attention has been given to the evolutionary role of gene loss. Gremlin 2 is a member of the DAN gene family and plays a significant role in tooth development by blocking the ligand-signaling pathway of BMP2 and BMP4. The goal of this study was to investigate the evolutionary history of gremlin 2 in cetartiodactyl mammals, a group that possesses highly divergent teeth morphology. Results from our analyses indicate that gremlin 2 has experienced a mixture of gene loss, gene duplication, and rate acceleration. Although the last common ancestor of cetartiodactyls possessed a single gene copy, pigs and camels are the only cetartiodactyl groups that have retained gremlin 2. According to the phyletic distribution of this gene and synteny analyses, we propose that gremlin 2 was lost in the common ancestor of ruminants and cetaceans between 56.3 and 63.5 million years ago as a product of a chromosomal rearrangement. Our analyses also indicate that the rate of evolution of gremlin 2 has been accelerated in the two groups that have retained this gene. Additionally, the lack of this gene could explain the high diversity of teeth among cetartiodactyl mammals; specifically, the presence of this gene could act as a biological constraint. Thus, our results support the notions that gene loss is a way to increase phenotypic diversity and that gremlin 2 is a dispensable gene, at least in cetartiodactyl mammals. 


\section{Research paper}

2

3

\section{Evolution of gremlin 2 in cetartiodactyl mammals: gene loss} coincides with lack of upper jaw incisors in ruminants

$7 \quad{ }^{1}$ Instituto de Ciencias Ambientales y Evolutivas, Facultad de Ciencias, Universidad Austral de

8 Chile, Valdivia, Chile. ${ }^{2}$ Unidad de Nefrología, Facultad de Medicina, Universidad Austral de

9 Chile, Valdivia, Chile. ${ }^{3}$ Instituto de Producción Animal, Facultad de Ciencias Agrarias,

\section{Corresponding author:}

14 Juan C. Opazo

15 Email: jopazo@gmail.com

16

17

18

19 


\section{Abstract}

21 Understanding the processes that give rise to genomic variability in extant species is an active

22 area of research within evolutionary biology. With the availability of whole genome sequences,

23 it is possible to quantify different forms of variability such as variation in gene copy number,

24 which has been described as an important source of genetic variability and in consequence of

25 phenotypic variability. Most of the research on this topic has been focused on understanding the

26 biological significance of gene duplication, and less attention has been given to the evolutionary

27 role of gene loss. Gremlin 2 is a member of the DAN gene family and plays a significant role in

28 tooth development by blocking the ligand-signaling pathway of BMP2 and BMP4. The goal of

29 this study was to investigate the evolutionary history of gremlin 2 in cetartiodactyl mammals, a

30 group that possesses highly divergent teeth morphology. Results from our analyses indicate that

31 gremlin 2 has experienced a mixture of gene loss, gene duplication, and rate acceleration.

32 Although the last common ancestor of cetartiodactyls possessed a single gene copy, pigs and

33 camels are the only cetartiodactyl groups that have retained gremlin 2 . According to the phyletic

34 distribution of this gene and synteny analyses, we propose that gremlin 2 was lost in the common

35 ancestor of ruminants and cetaceans between 56.3 and 63.5 million years ago as a product of a

36 chromosomal rearrangement. Our analyses also indicate that the rate of evolution of gremlin 2

37 has been accelerated in the two groups that have retained this gene. Additionally, the lack of this

38 gene could explain the high diversity of teeth among cetartiodactyl mammals; specifically, the

39 presence of this gene could act as a biological constraint. Thus, our results support the notions

40 that gene loss is a way to increase phenotypic diversity and that gremlin 2 is a dispensable gene,

41 at least in cetartiodactyl mammals. 


\section{Introduction}

43 One of the main goals of evolutionary biology is to understand the genetic basis of phenotypic

44 diversity. To address this question, scientists have made efforts to identify genes that are linked

45 to phenotypes and to explore the phenotypic consequences of genetic variability. With the

46 availability of whole genome sequences, it has been possible to compare different forms of

47 variability, and variation in gene copy number has been described as an important source of

48 genetic variability. To date, most of the research on this topic has been focused towards

49 understanding the biological significance of gene duplication, and less attention has been given

50 to the evolutionary role of gene loss (Olson, 1999; Albalat and Cañestro, 2016). In the literature,

51 there are examples of gene loss being associated with positive impacts on fitness. For example,

52 the loss of the CCR5 gene in humans is associated with resistance to AIDS (Dean et al., 1996),

53 and the loss of hair keratin genes in cetaceans is interpreted as an adaptation associated with the

54 transition from terrestrial to aquatic life (Nery et al., 2014; Yim et al., 2014). Thus, evolutionary

55 studies of genes that possess a clear link to a given phenotype represent an opportunity to

56 understand the phenotypic effects of gene loss and gene dispensability.

57 Gremlin 2, previously known as a protein related to Dan and Cerberus (PRDC), is a

58 member of the DAN gene family, a group of extracellular bone morphogenetic protein (BMP)

59 inhibitors, which was originally identified in a gene trap screen for developmentally significant

60 genes (Minabe-Saegusa et al., 1998). Gremlin 2, as an antagonist of BMPs (Kattamuri et al.,

61 2012), plays a role in several developmental processes including organogenesis, body patterning,

62 and tissue differentiation. In embryonic stages, this gene is expressed in the reproductive,

63 nervous, respiratory, musculoskeletal, and integumentary systems (Müller et al., 2006). 
64 Alternatively, during adulthood, it is a widely expressed gene found in high levels in ovaries, 65 brain, and spleen (Sudo et al., 2004).

In the literature, it has been shown that gremlin 2 interacts with BMP2 and BMP4 by

67 blocking their ligand-signaling pathway (Sudo et al., 2004). Human genetic studies have indicated that gremlin 2 variation can influence one's susceptibility of having a common tooth malformation (Kantaputra et al., 2015). Mutational analysis in seven out of 263 patients with different dental anomalies has revealed the presence of mutations predicted to cause disease.

Five patients of this study carried the same heterozygous mutations (Ala13Val) while the other two were carriers of two different heterozygous missense mutations (Gln76Glu and Glu136Asp) (Kantaputra et al., 2015). This genetic study supports the notion that inheritance of hypodontia is autosomal dominant, and this is related to gremlin 2. Despite this, the study also gives evidence of incomplete penetrance and variable expressivity. Genetic experiments provide further support for the role of gremlin 2 in tooth development (Brommage et al., 2014); it has been shown that gremlin 2 deficient mice have upper and lower incisor teeth with markedly reduced breadth and depth, and the upper incisors are more severely affected than lower ones (Vogel et al., 2015). According to Voget et al. (2015) no other significant phenotypic effects have been observed in grem $2^{--}$individuals, indicating that this gene could be dispensable. From a developmental perspective, it has been shown that the pathway that controls tooth differentiation is conserved in most mammals other than cetaceans, xenarthrans, and phocid seals (Armfield et al., 2013). In dolphins, it has been shown that expression of BMP4, which is one of the main targets of gremlin 2 (Sudo et al., 2004), is extended to the caudal region of the developing jaw, a region where the fibroblast growth factor 8 gene (FGF8) is express in most mammals (Armfield et al., 2013). This developmental difference could be related to the divergent dental phenotype of 
87 cetaceans. Similar results have been found during epibranchial placode development (Kriebitz et

88 al., 2009). Within the same mammalian clade, other groups also have different dental

89 morphologies. For example, ruminants do not possess incisors in the upper jaw; instead they

90 possess a dental pad. Canines are also absent in most ruminant species with the exception of elk

91 and red deer. This particular dental phenotype has consequences in the way these animals

92 process food, which is different compared to related species (herbivores) that possess incisors in 93 the upper jaw (e.g. horse). Finally, pangolins and baleen whales, both edentulous (i.e. toothless)

94 groups, represent the most extreme cases of dental modification in this group of mammals. The

95 lack of teeth in these groups has been related to the inactivation of tooth-specific genes (e.g.

96 C4orf26; Springer et al. 2016).

97 The main goal of this study was to investigate the evolutionary history of gremlin 2, a 98 gene that plays a significant role in the tooth development, in cetartiodactyl mammals a group 99 that possesses divergent tooth morphologies. Results from our analyses show that gremlin 2 has 100 experienced a mixture of gene loss, gene duplication, and rate acceleration. Although the last 101 common ancestor of cetartiodactyls possessed a single gene copy, pigs and camels are the only 102 cetartiodactyl groups that have retained gremlin 2. According to the phyletic distribution of this 103 gene and synteny analyses, we propose that gremlin 2 was lost in the common ancestor of 104 ruminants and cetaceans between 56.3 and 63.5 million years ago as a product of a chromosomal 105 rearrangement. Our analyses also indicate that the rate of evolution of gremlin 2 in pigs and 106 camels has been accelerated, and the possession of gremlin 2 clearly differentiates these groups 107 from all other cetartiodactyl mammals.

\section{Materials and methods}

109 DNA data collection and phylogenetic analyses 
110 We annotated gremlin 2 genes in representative species of laurasiatherian mammmals. Our study

111 included representative species from the orders Carnivora: cat (Felis catus), Siberian tiger

112 (Panthera tigris), dog (Canis familiaris), ferret (Mustela putorius), Weddell seal (Leptonychotes

113 weddellii), Pacific walrus (Odobenus rosmarus), panda (Ailuropoda melanoleuca);

114 Perissodactyla: Przewalski's horse (Equus ferus), horse (Equus caballus), donkey (Equus

115 asinus), Eulipotyphla: European hedgehog (Erinaceus europaeus); Chiroptera: Black flying fox

116 (Pteropus alecto), Large flying fox (Pteropus vampyrus), Egyptian fruit bat (Rousettus

117 aegyptiacus); Cetartiodactyla: pig (Sus Scrofa), alpaca (Vicugna pacos), dromedary (Camelus

118 dromedarius) and Bactrian camel (Camelus bactrianus); and Pholidota (Manis javanica)

119 (Supplementary Table S1). Mouse and kangaroo rat sequences were used as outgroups. Amino

120 acid sequences were aligned using the L-INS-i strategy from MAFFT v.6 (Katoh and Standley,

121 2013). Nucleotide alignment was generated using the amino acid alignment as a template using

122 the software PAL2NAL (Suyama et al., 2006). Phylogenetic relationships were estimated using

123 maximum likelihood and Bayesian approaches. We used the propose model tool of IQ-Tree

124 (Trifinopoulos et al., 2016) to select the best-fitting models for each codon position (TVMe+I,

$125 \mathrm{~K} 2 \mathrm{P}+\mathrm{G} 4$ and $\mathrm{HKY}+\mathrm{G} 4$, for first, second and third codon positions, respectively). We performed

126 a maximum likelihood analysis to obtain the best tree using the program IQ-Tree (Trifinopoulos

127 et al., 2016); and nodes support was assessed with 1000 bootstrap pseudoreplicates using the

128 ultrafast routine. Bayesian searches were conducted in MrBayes v.3.1.2 (Ronquist and

129 Huelsenbeck, 2003); two independent runs of six simultaneous chains for $20 \times 10^{6}$ generations

130 were set, and every 2,500 generations were sampled using default priors. The run was considered

131 to have reached convergence once the likelihood scores reached an asymptote and the average

132 standard deviation of the split frequencies remained $<0.01$. We discarded all trees that were 
133 sampled before convergence, and we evaluated support for the nodes and parameter estimates

134 from a majority rule consensus of the last 4,000 trees.

135

136 Assessments of Conserved Synteny

137 We examined genes found up- and downstream of gremlin 2 in the laurasiatherian mammal

138 representative species. Synteny analyses were conducted for dog (Canis familiaris), panda

139 (Ailuropoda melanoleuca), horse (Equus caballus), donkey (Equus asinus), European hedgehog

140 (Erinaceus europaeus), Large flying fox (Pteropus vampyrus), Egyptian fruit bat (Rousettus

141 aegyptiacus), alpaca (Vicugna pacos), dromedary (Camelus dromedarius), pig (Sus scrofa),

142 sheep (Ovis aries), goat (Capra hircus), cow (Bos taurus), minke whale (Balaenoptera

143 acutorostrata), killer whale (Orcinus orca), baiji (Lipotes vexillifer), and Malayan pangolin

144 (Manis javanica). Initial ortholog predictions were derived from the EnsemblCompara database

145 (Herrero et al., 2016) and were visualized using the program Genomicus v85.01 (Muffato et al.,

146 2010). In other cases, the genome data viewer platform from the National Center for

147 Biotechnology information was used.

148

149 Results and Discussion

150 Gremlin 2 is a protein-coding gene located on the reverse strand that has two exons, where the

151 first is the one that possesses the information for the final protein. In most laurasitherian

152 mammals and humans the length of the coding portion of the gene (507 bp) is well conserved

153 while some variation is found in cetartiodactyl mammals.

154

\section{Phylogenetic relationships}


156 We constructed a phylogenetic tree in which we included representative species of

157 laurasiatherian mammals (Fig. 1). Our phylogenetic analysis recovered the monophyly of each

158 laurasiatherian order included in our sampling (Fig. 1). Although the phylogenetic relationships

159 among laurasiatherian mammals at the ordinal level are still a matter of debate, the most

160 important departures from current hypotheses detected here was the sister group relationship

161 between Eulipotyphla and Carnivora (Fig. 1) and the sister group relationship between Pholidota

162 and Cetartiodactyla. Specifically, in most studies, eulipotyphlan species appear sister to all other

163 laurasiatherian mammals (Nery et al., 2012; Foley et al., 2016), and Pholidota is recovered as

164 sister to Carnivora (Meredith et al. 2011, Du Toit et al 2014). The synteny analysis provided

165 further support for the identity of the gremlin 2 gene lineage in this group of mammals (Fig. 2);

166 genes found downstream were well conserved in all examined species (Fig. 2). According to our

167 survey, most species included in this study possessed four downstream genes ( $R G S 7, F H, K M O$

168 and $O P N 3$ ) that define the identity of this genomic region (Fig. 2). Although the genes found

169 upstream were more variable, they were to some degree more conserved in the different groups

170 (Fig. 2). For example, in both camelid species four upstream genes (RNF2, TRMT1L, SWT1 and

$171 I V N S 1 A B P$ ) were detected that were well conserved (Fig. 2). Similar results were found for

172 sheep, goat, cow, minke whale, killer whale, and baiji (Fig. 2).

173

174 Molecular rates in cetartiodactyls and pholidotans

175 The rate of molecular evolution, as measured here using branch lengths, was variable (Fig. 1),

176 though the most striking result was that of the accelerated evolution of cetartiodactyls and to a

177 lesser extent that of pholidotans (Fig. 1). To test whether the rate of gremlin 2 evolution in these

178 groups of species is significantly higher, we performed a relative rate test (Tajima, 1989) using 
179 the software MEGA 7 (Kumar et al., 2016). We compared the rate of evolution of cetartiodactyl

180 and pholidotan sequences using the cat sequence as a reference and the mouse sequence as the

181 outgroup. Results of this analysis confirmed what was observed in our phylogenetic tree, i.e. the

182 rate of evolution of cetartiodactyls and pholidotans is significantly higher than that of other

183 laurasiatherian mammals (Supplementary table S2). This rate acceleration seems to be specific to

184 this locus, since the same test in other members of the gene family (GREM1, CERI and DAND5)

185 did not reveal evidence of rate acceleration in these groups (Supplementary table S3). To further

186 investigate the evolutionary pattern of gremlin 2 in cetartiodactyls and pholidotans, we made an

187 amino acid alignment that included, in addition to the cetartiodactyl and pholidotan sequences,

188 representative species of the laurasiatherian orders Perissodactyla, Carnivora, Chiroptera, and

189 Eulipotyphla. From this, we found that there are 13 synapomorphies that define the gremlin 2

190 genes in cetartiodactyls (Fig. 3). Among these, we identified 11 amino acid changes and two

191 deletions (Fig. 3). Of all of the amino acid substitutions, changes at positions 34 (Tyr to Arg),

192109 (His to Pro), 131 (Thr to Ala), and 132 (Ser to Ala) represent changes affecting

193 hydrophobicity (Fig. 3). Of the 13 synapomophies observed in cetartiodactyls, only one is shared

194 with pholidotans (Ser165Gly)(Fig. 3). Amino acid 110 also represents a synapomorphy in

195 pangolins however the amino acid identity is different from that of cetartiodactyls (Fig. 3).

196

197 Gene copy number variation and differential retention in cetartiodactyls

198 Most laurasiatherian species possess a single copy of the gene with the exception of pig (Sus

199 scrofa) that has two copies on chromosome 10 (Fig. 5). As in all examined species, in pig, one of

200 the copies (gremlin 2-T1) was found on the 5' side of the regulator of the G-protein signaling 7

201 gene (RGS7) (Fig. 2). The second copy (gremlin 2-T2) was found within the RGS7 gene, 
202 specifically between exons 13 and 14 (Fig. 4). At the amino acid level both copies differed in

203 one amino acid (position 155); gremlin 2-T1 possessed an arginine, and gremlin 2-T2 possessed 204 a lysine.

205 Among cetartiodactyls, we observed that gremlin 2 was differentially retained (Fig. 2).

206 Species belonging to the suborders Tylopoda (the group that includes camels, alpacas, vicuñas, 207 and guanacos) and Suiformes (the group that includes pigs and peccaries) were the only groups 208 in which gremlin 2 was present (Fig. 2). In cetaceans and ruminants, gremlin 2 was not present. 209 Thus, according to the phyletic distribution of gremlin 2 within the main groups of 210 cetartiodactyls, the most likely scenario is that the deletion of the gene occurred between 56.3 211 and 63.5 million of years ago in the common ancestor of the clade that includes ruminants, 212 hippopotamuses, and cetaceans (Fig. 5). However, until information regarding gremlin 2 in 213 hippopotamuses is obtained, caution must be taken when interpreting these results. If, in the 214 future, the hippopotamus genome is found to possess gremlin 2, we can determine that two 215 independent gene losses occurred, one in the ancestor of ruminants and a second in the ancestor 216 of cetaceans. For now, a single gene loss event is assumed.

217 To gain insight into the genetic mechanisms that gave rise to the deletion of gremlin 2, 218 we compared the chromosomal location of genes found up- and downstream of gremlin 2 in 219 human, cow, and sheep (Fig. 6). We identified a chromosomal region of approximately $12 \mathrm{Mb}$, 220 which in human was on the 5' side of gremlin 2 (Fig. 6; pink box), while in cow and sheep it was 221 found in a different chromosome in relation to other genes that are linked to gremlin 2 (Fig. 6; 222 pink box). In cow, this region was moved from chromosome 16 to 28 , while in sheep it was 223 moved from chromosome 12 to 25 (Fig. 6). As a consequence of this chromosomal 224 rearrangement, the regions that are located up- and downstream of the chromosome piece that 
225 was moved are now located together in both cow and sheep (Fig. 6). Thus, in these species, the

226 gene that is found on the 5' side of gremlin 2 (FMN2) was part of the chromosomal block that

227 was moved to a different chromosome (Fig. 6) whereas the gene located on the 3' side (RGS7)

228 was not. From this, we suggest that one of the break points that gave rise to the chromosomal

229 rearrangement was the chromosomal region where gremlin 2 was located (Fig. 6). Information

230 regarding the chromosomal location of genes found up- and downstream of gremlin 2 in

231 cetaceans would be an important piece of information not only to understand the genetic

232 mechanisms responsible for the deletion of gremlin 2, but also to shed light on the number of

233 gene loss events that have occurred in the clade including cetaceans, hippopotamuses and

234 ruminants. Thus, if cetaceans and ruminants show similar patterns, we could speculate that this

235 genetic event occurred in the last common ancestor of the group and was inherited by all

236 descendant lineages (cetaceans, hippopotamuses, and ruminants). However, if cetaceans and

237 ruminants show different patterns, we can postulate two deletion events. Preliminary information

238 from baiji shows a similar pattern to that seen in ruminants, supporting the hypothesis that one

239 gene loss occurred in the last common ancestor of cetaceans, hippopotamuses, and ruminants

240 (Supplementary fig. 1).

241 From a biomedical perspective, the loss of gremlin 2 (e.g. in cow, sheep, goat, dolphins,

242 whales) represents a natural gene knockout (evolutionary mutant models according to (Albertson

243 et al., 2009), thus presenting an outstanding opportunity to understand gremlin 2 biology. From a

244 physiological standpoint, this phenomenon is interesting as gremlin 2 plays a role in several

245 developmental processes, including organogenesis, body patterning, and tissue differentiation.

246 Thus, several questions regarding the mode of action of this gene could be investigated

247 considering the lack of this gene in certain species. For example, what happens with BMP2 and 
248 BMP4 in the absence of gremlin 2? Are these BMPs free of any antagonist action? Or does

249 another member of the DAN gene family fulfill gremlin 2's molecular role? From a phenotypic

250 perspective, it has been shown that BMP2 and BMP4 are involved in the signaling pathway that

251 regulates tooth development (Aberg et al., 1997; Nadiri et al., 2004). Genetic manipulation

252 experiments have shown that gremlin 2 deficient mice have upper and lower incisor teeth with

253 markedly reduced breadth and depth, and the upper incisors are more severely affected than the

254 lower ones (Kantaputra et al., 2015; Vogel et al., 2015). This supports the argument that the lack

255 of gremlin 2 contributes to the divergent dental phenotype of ruminants and cetaceans.

256 Ruminants do not have incisors in the upper jaw; instead they have a dental pad. With the

257 exception of elk and red deer, canines are also absent in most species. This particular dental

258 phenotype affects how ruminants eat, which differ from phylogenetically related species that

259 have incisors in the upper jaw (e.g. horse). For example, cows use their tongue to wrap and pull

260 leaves into their mouths between the incisors of the lower jaw and the dental pad; thus, plants are

261 not clearly cut during feeding. This contrasts with the feeding method of phylogenetically related

262 species that have upper and lower incisors; these species cut plants and graze deeply. Once the

263 food is in their mouths, cows swing their heads to chew the food slightly and mix it with saliva

264 before swallowing. This lateral chewing action is required to cut plant tissues because molars

265 and premolars of the maxillary jaw are wider than those located on the mandibular jaw.

266 Conversely, sheep use their lips and teeth as their primary tools for food prehension. Their lips

267 are used to bring food into their mouths, and the incisors of the lower jaw in combination with

268 the dental pad allow them to cut leaves. As a consequence, sheep can bite closer to the ground

269 and have the ability to be more selective. 
271 subgroup (toothed whales) has teeth while another subgroup (baleen whales) does not. To further

272 complicate this scenario, it has been argued that it is impossible to define teeth homology

273 between toothed whales and non-cetacean mammals (Armfield et al., 2013). From a

274 developmental perspective, it has been demonstrated that the pathway that controls tooth

275 differentiation and number in cetaceans is different from the typical mammalian pattern

276 (Armfield et al., 2013). Particularly interesting is that the expression pattern of BMP4, one of the

277 main targets of gremlin 2, differs between cetaceans and non-cetacean mammals (Sudo et al.,

278 2004). The case of hippopotamuses remains an open question until the genome is sequenced.

279 However, we can speculate that, as has been shown in cetaceans, the tooth morphology of this

280 group could be related to different regulatory pathways controlling teeth development as a

281 consequence of the absence gremlin 2.

282 Finally, the fact that pangolins, a group of species that do not have teeth, possess a putatively

283 functional copy of gremlin 2 highlights that the toothless phenotype has been achieved by

284 genetic modifications in tooth-specific genes (e.g. C4orf26; Springer et al., 2016). The presence

285 of toothless species that possess (e.g. pangolins) and do not possess (e.g. baleen whales) gremlin

2862 supports this idea. Following the same argument, and given the relationship between gremlin 2

287 and upper jaw incisor development (Kantaputra et al., 2015; Vogel et al., 2015), we should

288 expect that species that lack upper jaw incisors (e.g. ruminants) but retain all other teeth should

289 not share the modification in tooth-specific genes present in toothless species (Springer et al.,

290 2016). Outside Laurasiatheria, armadillos could be seen as an exception to this as they do not

291 possess incisors but they have a putatively functional gremlin 2 gene. However, it has been 
292 described that armadillos have four to six primordial incisors at birth, which means that incisors

293 are developed during embryogenesis but are absorbed shortly after birth (Capizzo et al. 2013).

294

295 Concluding remarks

296 Our results show that in cetartiodatyl mammals gremlin 2 has experienced a mixture of gene

297 loss, gene duplication, and rate acceleration. Although the last common ancestor of

298 cetartiodactyls possessed a single copy of the gene, species belonging to the suborders Tylopoda

299 (the group that includes camels, alpacas, vicuñas, and guanacos) and Suiformes (the group that

300 includes pigs and peccaries) are the only groups that have retained gremlin 2 (Fig. 5). These

301 groups also experienced acceleration in the rate of evolution of this gene, and it is this that

302 clearly differentiates them from all other laurasiatherians (Fig. 3). The fact that all amino acid

303 changes that define the gremlin 2 gene in Tylopoda and Suiformes are present in both groups

304 suggests that this gene and its corresponding protein were remodeled in the last common

305 ancestor of cetartiodactyls and subsequently inherited by all descendant lineages (Fig. 5). After

306 that, gremlin 2 was probably lost in the ancestor of ruminants, hippopotamuses, and cetaceans

307 between 56.3 and 63.5 million of years ago (Fig. 5). By removing a biological constraint

308 imposed by the presence of gremlin 2, the lack of this gene could explain teeth diversity in these

309 groups of mammals. Thus, the results presented here support the argument that gene loss is a

310 way to increase phenotypic diversity (Olson, 1999; Albalat and Cañestro, 2016) and that gremlin

3112 is a dispensable gene at least in this group of mammals. 


\section{Figure legends}

313

314 Figure 1. Maximum likelihood phylogenetic tree depicting relationships among gremlin 2 genes

315 in laurasiatherian mammals. Numbers on the nodes correspond to Bayesian posterior

316 probabilities and maximum likelihood bootstrap support values. Sequences of mouse and

317 kangaroo rat were used as outgroups.

318

319 Figure 2. Patterns of conserved synteny in the genomic regions that harbor gremlin 2 genes in

320 laurasiatherian mammals. Upper panel: genomic region that harbors gremlin 2 genes. Lower

321 panel: conserved synteny in the genomic region that would be the putative location of the

322 gremlin 2 gene in ruminants and cetaceans.

323

324 Figure 3. An alignment of gremlin 2 amino acid sequences from laurasiatherian mammals.

325 Amino acid positions in bold denote the 11 amino acid synapomorphies that define the sequences 326 of pigs and camels.

327

328 Figure 4. Schematic representation of the gremlin 2 syntenic region in pigs. One of the copies

329 (gremlin 2-T1) is located on the 5' side of the regulator of the G-protein signaling 7 gene (RGS7)

330 whereas the second copy (gremlin 2-T2) is located within the RGS7 gene, specifically between 331 exons 13 and 14.

333 Figure 5. An evolutionary hypothesis regarding the evolution of the gremlin 2 gene in 334 cetartiodactyl mammals. According to this model, the last common ancestor of cetartiodactyls 
335 possessed a single copy of the gene. Species belonging to the suborders Tylopoda, the group that

336 includes camels, alpacas, vicuñas and guanacos, and Suiformes, the group that includes pigs and

337 peccaries, were the only groups that retained gremlin 2 . According to the phyletic distribution of

338 gremlin 2, we propose that this gene was lost in the common ancestor of ruminants,

339 hippopotamuses, and cetaceans between 56.3 and 63.5 million of years ago as a product of a

340 chromosomal rearrangement.

341

342 Figure 6. Schematic representation of the chromosomal regions that harbor genes located up-

343 and downstream of gremlin 2. Upper panel: chromosomal region that harbors genes that are up-

344 and downstream of gremlin 2 in humans. Middle panel: Chromosomal regions (chrs 16 and 28)

345 that harbor genes that are located up- and downstream of the putative location of gremlin 2 in

346 cow. Lower panel: Chromosomal regions (chrs 25 and 12) that harbor genes that are located up-

347 and downstream of the putative location of gremlin 2 in sheep. 


\section{References}

350

351

354

355

356

357

358

359

360

361

362

363

364

365

366

367

368

369

370

371
Aberg, T., Wozney, J. and Thesleff, I., 1997. Expression patterns of bone morphogenetic proteins (Bmps) in the developing mouse tooth suggest roles in morphogenesis and cell differentiation. Dev Dyn 210, 383-96.

Albalat, R. and Cañestro, C., 2016. Evolution by gene loss. Nat Rev Genet 17, 379-91.

Albertson, R.C., Cresko, W., Detrich, H.W. and Postlethwait, J.H., 2009. Evolutionary mutant models for human disease. Trends Genet 25, 74-81.

Armfield, B.A., Zheng, Z., Bajpai, S., Vinyard, C.J. and Thewissen, J., 2013. Development and evolution of the unique cetacean dentition. PeerJ 1, e24.

Brommage, R., Liu, J., Hansen, G.M., Kirkpatrick, L.L., Potter, D.G., Sands, A.T., Zambrowicz, B., Powell, D.R. and Vogel, P., 2014. High-throughput screening of mouse gene knockouts identifies established and novel skeletal phenotypes. Bone Res 2, 14034.

Capizzo, A., Moses, E., Shirley, E. and Myers, P., 2013. "Dasypodidae" (On-line), Animal Diversity Web. Available at http://animaldiversity.org/accounts/Dasypodidae/ (accessed November 30, 2016 ).

Dean, M., Carrington, M., Winkler, C., Huttley, G.A., Smith, M.W., Allikmets, R., Goedert, J.J., Buchbinder, S.P., Vittinghoff, E., Gomperts, E., Donfield, S., Vlahov, D., Kaslow, R., Saah, A., Rinaldo, C., Detels, R. and O'Brien, S.J., 1996. Genetic restriction of HIV-1 infection and progression to AIDS by a deletion allele of the CKR5 structural gene. Hemophilia Growth and Development Study, Multicenter AIDS Cohort Study, Multicenter Hemophilia Cohort Study, San Francisco City Cohort, ALIVE Study. Science 273, 1856-62. 
372 Du Toit, Z., Grobler, J.P., Kotzé, A., Jansen, R., Brettschneider, H. and Dalton, D.L., 2014. The

373

374

375

376

377

378

379

380

381

382

383

384

385

386

387

388

389

390

391

392

393

complete mitochondrial genome of temminck's ground pangolin (Smutsia temminckii;

Smuts, 1832) and phylogenetic position of the Pholidota (Weber, 1904). Gene 551, 49-

54.

Foley, N.M., Springer, M.S. and Teeling, E.C., 2016. Mammal madness: is the mammal tree of life not yet resolved? Philos Trans R Soc Lond B Biol Sci 371.

Herrero, J., Muffato, M., Beal, K., Fitzgerald, S., Gordon, L., Pignatelli, M., Vilella, A.J., Searle, S.M., Amode, R., Brent, S., Spooner, W., Kulesha, E., Yates, A. and Flicek, P., 2016. Ensembl comparative genomics resources. Database (Oxford) 2016.

Kantaputra, P.N., Kaewgahya, M., Hatsadaloi, A., Vogel, P., Kawasaki, K., Ohazama, A. and Ketudat Cairns, J.R., 2015. GREMLIN 2 Mutations and Dental Anomalies. J Dent Res $94,1646-52$.

Katoh, K. and Standley, D.M., 2013. MAFFT multiple sequence alignment software version 7: improvements in performance and usability. Mol Biol Evol 30, 772-80.

Kattamuri, C., Luedeke, D.M., Nolan, K., Rankin, S.A., Greis, K.D., Zorn, A.M. and Thompson, T.B., 2012. Members of the DAN family are BMP antagonists that form highly stable noncovalent dimers. J Mol Biol 424, 313-27.

Kriebitz, N.N., Kiecker, C., McCormick, L., Lumsden, A., Graham, A. and Bell, E., 2009. PRDC regulates placode neurogenesis in chick by modulating BMP signalling. Dev Biol 336, 280-92.

Kumar, S., Stecher, G. and Tamura, K., 2016. MEGA7: Molecular Evolutionary Genetics Analysis Version 7.0 for Bigger Datasets. Mol Biol Evol 33, 1870-4. 
394 Meredith, R.W., Janecka, J.E., Gatesy, J., Ryder, O.A., Fisher, C.A., Teeling, E.C., Goodbla, A., 395 Eizirik, E., Simao, T.L.L., Stadler, T., Rabosky, D.L., Honeycutt, R.L., Flynn, J.J, 396 Ingram, C.M., Steiner,C., Williams, T.L., Robinson, T.J., Burk-Herrick, A., Westerman, M., Ayoub, N.A., Springer, M.S. and Murphy, W.J. 2011. Impacts of the Cretaceous Terrestrial Revolution and KPg Extinction on Mammal Diversification. Science 334, 521-524.

Minabe-Saegusa, C., Saegusa, H., Tsukahara, M. and Noguchi, S., 1998. Sequence and expression of a novel mouse gene PRDC (protein related to DAN and cerberus) identified by a gene trap approach. Dev Growth Differ 40, 343-53.

Muffato, M., Louis, A., Poisnel, C.E. and Roest Crollius, H., 2010. Genomicus: a database and a 404 browser to study gene synteny in modern and ancestral genomes. Bioinformatics 26, 405 $1119-21$.

Müller, I.I., Knapik, E.W. and Hatzopoulos, A.K., 2006. Expression of the protein related to Dan and Cerberus gene--prdc--During eye, pharyngeal arch, somite, and swim bladder development in zebrafish. Dev Dyn 235, 2881-8.

409

410

Nadiri, A., Kuchler-Bopp, S., Haikel, Y. and Lesot, H., 2004. Immunolocalization of BMP-2/-4, FGF-4, and WNT10b in the developing mouse first lower molar. J Histochem Cytochem 52, 103-12.

Nery, M.F., Arroyo, J.I. and Opazo, J.C., 2014. Increased rate of hair keratin gene loss in the cetacean lineage. BMC Genomics 15, 869.

Nery, M.F., Gonzalez, D.J., Hoffmann, F.G. and Opazo, J.C., 2012. Resolution of the laurasiatherian phylogeny: Evidence from genomic data. Molecular Phylogenetics and Evolution 64, 685-689. 
417 Olson, M.V., 1999. When less is more: gene loss as an engine of evolutionary change. Am J $418 \quad$ Hum Genet 64, 18-23.

419 Ronquist, F. and Huelsenbeck, J.P., 2003. MrBayes 3: Bayesian phylogenetic inference under $420 \quad$ mixed models. Bioinformatics 19, 1572-1574.

421 Springer, M.S., Starrett, J., Morin, P.A., lanzetti, A., Hayashi, C. and Gatesy, J, 2016.

422 Inactivation of C4orf26 in toothless placental mammals. Mol Phylogenet Evol 95, 34-45.

423 Sudo, S., Avsian-Kretchmer, O., Wang, L.S. and Hsueh, A.J., 2004. Protein related to DAN and

424 cerberus is a bone morphogenetic protein antagonist that participates in ovarian paracrine 425 regulation. J Biol Chem 279, 23134-41.

426 Suyama, M., Torrents, D. and Bork, P., 2006. PAL2NAL: robust conversion of protein sequence 427 alignments into the corresponding codon alignments. Nucleic Acids Res 34, W609-12.

Tajima, F., 1989. Statistical method for testing the neutral mutation hypothesis by DNA

430

431

432

433

434 435 436 437 438 439 polymorphism. Genetics 123, 585-95.

Trifinopoulos, J., Nguyen, L.T., von Haeseler, A., Minh, B.Q. 2016. W-IQ-TREE: a fast online phylogenetic tool for maximum likelihood analysis. Nucleic Acids Res. 44, W232W235.

Vogel, P., Liu, J., Platt, K.A., Read, R.W., Thiel, M., Vance, R.B. and Brommage, R., 2015. Malformation of incisor teeth in Grem2 $2^{-{ }^{-}}$mice. Vet Pathol 52, 224-9.

Yim, H.S., Cho, Y.S., Guang, X., Kang, S.G., Jeong, J.Y., Cha, S.S., Oh, H.M., Lee, J.H., Yang, E.C., Kwon, K.K., Kim, Y.J., Kim, T.W., Kim, W., Jeon, J.H., Kim, S.J., Choi, D.H., Jho, S., Kim, H.M., Ko, J., Kim, H., Shin, Y.A., Jung, H.J., Zheng, Y., Wang, Z., Chen, Y., Chen, M., Jiang, A., Li, E., Zhang, S., Hou, H., Kim, T.H., Yu, L., Liu, S., Ahn, K., Cooper, J., Park, S.G., Hong, C.P., Jin, W., Kim, H.S., Park, C., Lee, K., Chun, S., 

aquatic adaptation in cetaceans. Nat Genet 46, 88-92. 


\section{Figure 1 (on next page)}

Maximum likelihood phylogenetic tree depicting relationships among gremlin 2 genes in laurasiatherian mammals

Figure 1. Maximum likelihood phylogenetic tree depicting relationships among gremlin 2 genes in laurasiatherian mammals. Numbers on the nodes correspond to Bayesian posterior probabilities and maximum likelihood bootstrap support values. Sequences of mouse and kangaroo rat were used as outgroups. 


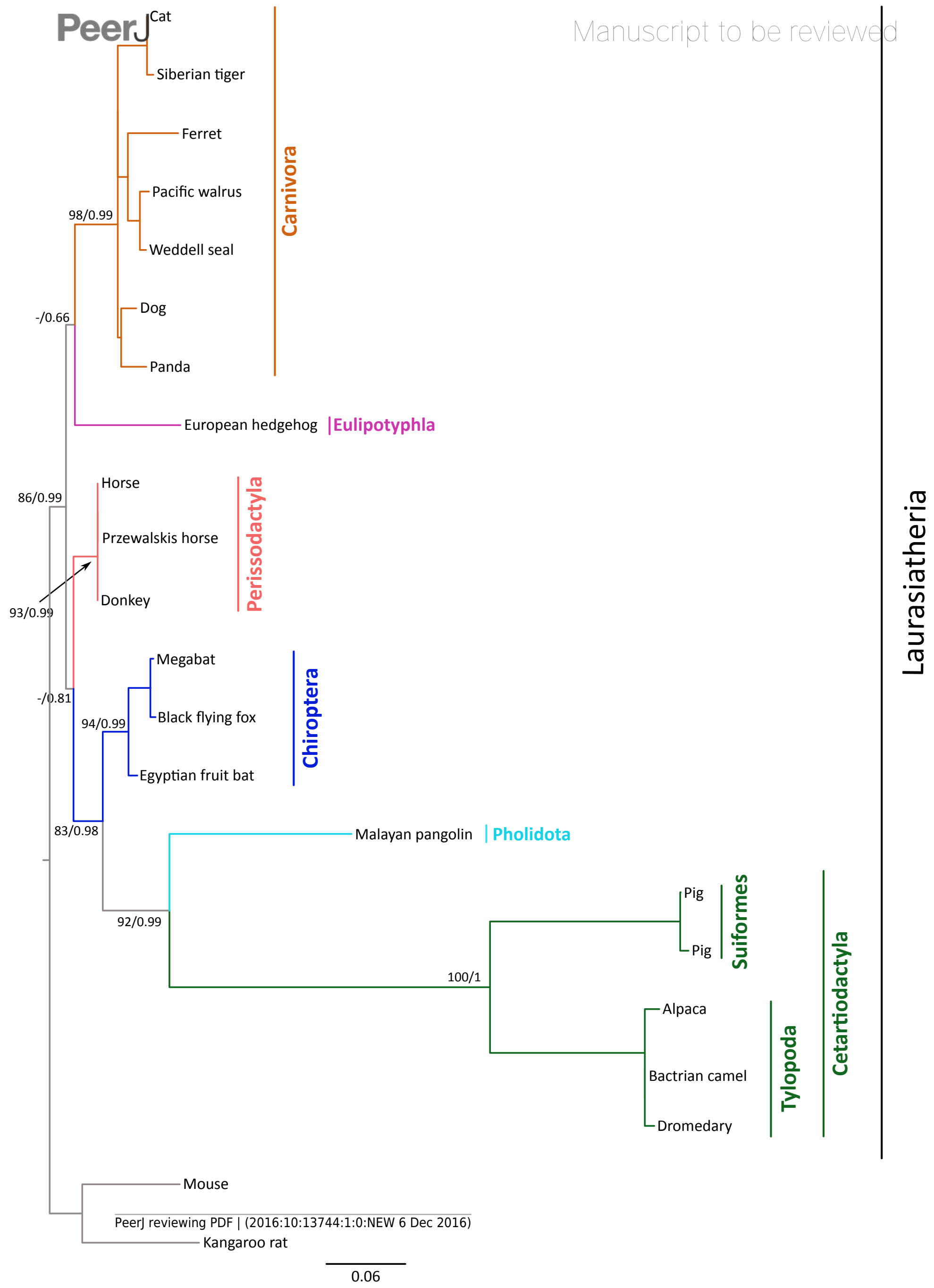


Figure 2 (on next page)

Patterns of conserved synteny in the genomic regions that harbor gremlin 2 genes in laurasiatherian mammals

Figure 2. Patterns of conserved synteny in the genomic regions that harbor gremlin 2 genes in laurasiatherian mammals. Upper panel: genomic region that harbors gremlin 2 genes. Lower panel: conserved synteny in the genomic region that would be the putative location of the gremlin 2 gene in ruminants and cetaceans. 


\section{PeerJ}
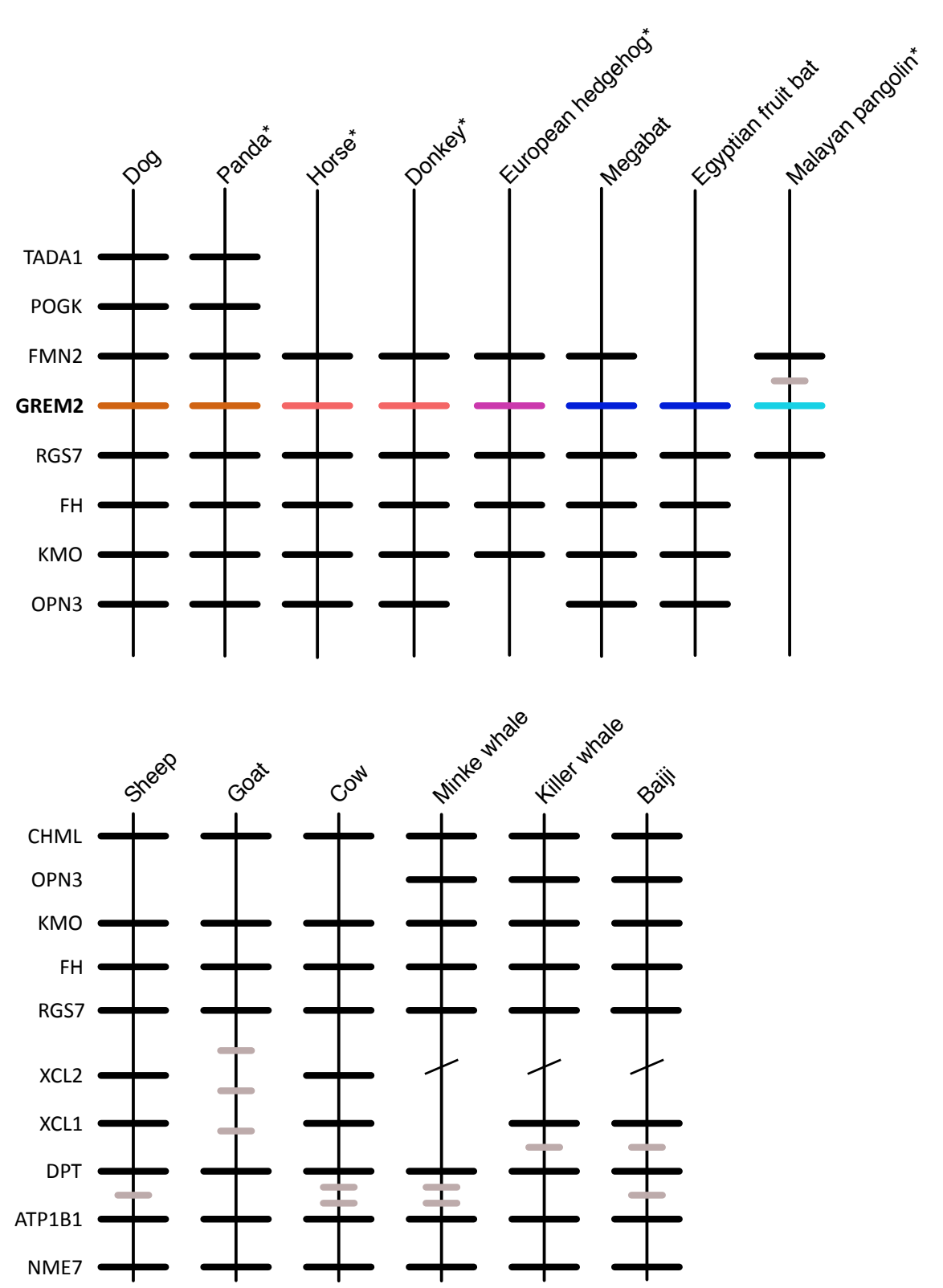

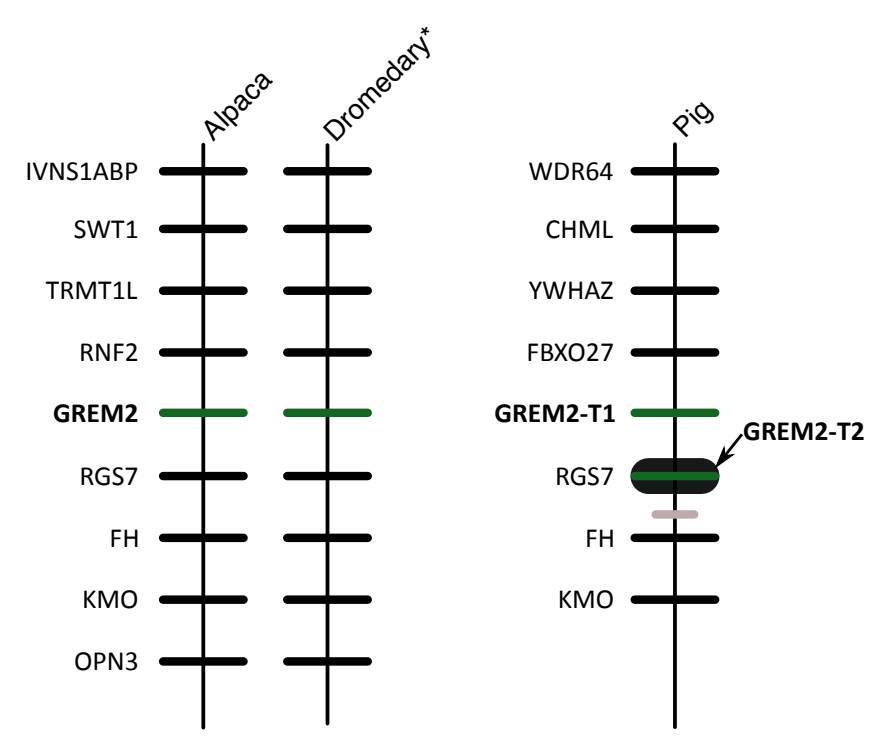




\section{Figure 3 (on next page)}

An alignment of gremlin 2 amino acid sequences from laurasiatherian mammals

Figure 3. An alignment of gremlin 2 amino acid sequences from laurasiatherian mammals. Amino acid positions in bold denote the 11 amino acid synapomorphies that define the sequences of pigs and camels. 
Alpaca

Bactrian camel

Dromedary

Pig

Pig

Malayan pangolin

Siberian tiger

Cat

Dog

Ferret

Panda

Pacific walrus

Weddell seal

European hedgehog

Horse

Donkey

Przewalski horse

Black flying fox

Egyptian fruit bat

Megabat
40

50

60

70

80

90

MFWKISWSILLAAL GKLEEIQGARPAGAI PSPRKDRGTNNSQNW QH-IREVLSSSQEAL VVTERRYLRRWCKT ORLRQTVREEGCHSR FWKLSVSLLLMAAL EKVEDAORARPAGSI PSPRKDGSPESTGRW OL-IKEVLASSOEAL VVTERRYLRDWCKT OPLROTVHEEGCHSR TH MLRRLSLSVLLVAVL VEAAGARKARPAGAI PSPYKDGGSNASERW HHQVKEVLASSHEAL VVTERKYKSDWCKT QPLRQTVGEEGCRS MFWKLSLLCLVAVL VKAAEARKNRAGA PSPYKDGSSHSERW QHQIKEVLASSQEAL VVTERKYKSDWCKT QPLRQTVSEEGCRSR MFWKLSLSLCLVAVL VKVAEARKNRPAGA PSPYKDGSSNHERW OHOIKEVLASSOEAL VVTERKYLKSDWCKT OPLROTVSEEGCRSR MTHTISTSICIVAVT VZTATARTHR MFWKLSLSLCLVAVL VKAAEARKNRPAGAI PSPYK-GSSNHSERW QHQIKEVLASSQEAL VVTERKYLRDWCKT QPLRQTVSEDGCRSR MFWKLSLLCLVAVL VKVAEARKNRAGA PSPYKDGSSHSERW QQQIKEVLASSQEAL VVTERKYLSDWCKT QPLRQTVSEEGCRSR MFWKLSLSLCLVAVL VKVAEARKNRPAGA PSPYKDGSSNHERW QHQIKEVLASSQEAL VVTERKYLSDWCKT QPLRQTVSEEGCRSR MFWKLSLSLCLVAVL VKVAEARKNRPAGA PSPYKDGSSNHSERW OHOTKEVLASSOEAL VVTERKYIKSDWCKT OPLROTVSEFGCRSR

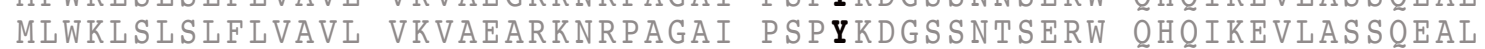
MLWKLLSLFLVAVL VKVAEARKNRPAGI PSPYKDGSSNTERW QHQIKEVLASSQEAL MLWKLSLSLFLVAVL VKVAEARKNRPAGA PSPYKDGSSNTSERW QHQIKEVLASSQEAL MFWKLSISLILVAVI LKVGDARKNRPAGAI PSPYKDGSSNTSERW OHOIKEVLASSOEAL MFWKLSLLLLVAVL VKVVDARKNRPAGA PSPYKDGSNTSERW QHQIKEVLASSQEAL MFWKLSLSLLLVAVL VKVGDARKRPAGAI PSPYKDGSNTSERW QHQIKEVLASSQEA VTERKYTKSDWCKT VVTERKYLKSDWCKT

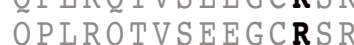
VTERKYLKSDWCKT OPLROTVSETGCRSR VVTERKYLKSDWCKT OPLROTVGEEGCRSR VVTERKYLRSDWCT QPLRQTVGEEGCRSR
110

TVLNHFCYGOCNSFY IPRPGGEGEGSGSF TVLNHFCYGOCNSFY IPRPGGEGEGSGSFO TVLNHFCYGQCTSSH TPRPGGEGEGSGSF 0 TVLNRFCYGQCNSFF IPRPGGGSW--GSFQ TVLNRFCYGOCNSFF IPRPGGGSW--GSFO TVLNRFCYGOCNSFY IPRHARAEE--GSFO TILNRFCYGOCNSFY T I L NRF C Y G Q C N S F Y T I LNRF CYGQCNSFY TVLNRF C Y GQC N S F Y I INRF CYGOCNSFY I L NRF CYG OCN S FY T I L NRF C Y GQCN S F Y T I LNRF CYGQCNS FY T I L N R F C Y GOCNS F Y TINRFCYGOCNSF T I L NRF C Y G Q C N S F Y TVLNRFCYGQCNSFY TVLNRF CYGQCNSFY TVLNRFCYGOCNSFY
130

140

1,50

160

1,70

作 CRPORAAALLV ELOCPSRDPPVRLRK IOKVKOCRCMSVNLG ADESSCAFCRPQRAAALLV ELQCPSRDPPVRLRK IQKVKQCRCMSVNLG ADES $\begin{array}{llll}\text { SCAFCRPQRAAALLV } & \text { ELQCPGRDPPFHLRK } & \text { IQKVKQCRCMSVTLG } & \text { SDEP- }\end{array}$ SCAFCRPORAAALLV ELOCPGRDPPEHLRK IOKVKOCKCMSVTLG SDEP-

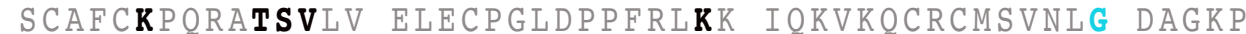

$\begin{array}{lllll}P R H V R K E E--E S F Q & \text { SCAFCKPQRVTSVLV } & \text { ELECPGLDPPFRLKK } & \text { I QKVKQCRCMSVNLS } & \text { DSDKQ } \\ \text { PRHVRKEE--ESFQ } & \text { SCAFCKPQRVTSVLV } & \text { ELECPGLDPPFRLKK } & \text { I QKVKQCRCMSVNLS DSDKQ }\end{array}$ $\begin{array}{llll}\text { IPRVRKEE--ESFQ } & \text { SCAFCKPQRVTSVLV } & \text { ELECPGLDPPFRLKK IQKVKQCRCMSVNLS DSDKO }\end{array}$ $\begin{array}{llll}P R \mathbf{H} R K E--E S F Q & \text { SCAFCKPQRVTSVLV ELEPGMDPFRLKK } & \text { IQKVKQCRCMSVNLS DSDQ }\end{array}$ $\begin{array}{llll}\text { RHVRKEE--ESFO SCAFCKPORVTSVLV } & \text { ELECPGLDPPFRLKK IOKVKOCRCMSVNLS DSDKO }\end{array}$ $\begin{array}{llll}P R \mathbf{H} R E E--E S F Q & \text { SCAFCKPQRVTSVLV } & \text { ELECPGMDPPFRLKK IQKVKQCRCMSVNLS DSDKQ }\end{array}$

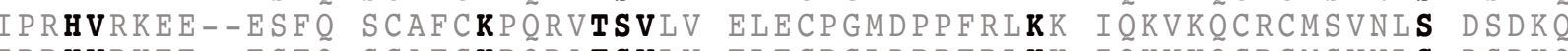
PRHVRKEE--ESFO SCAFCKPQRATSVLV ELECPGLDPPFRLKK IOKVKOCRCMSVNLS DSDKO $\begin{array}{lll}P R \boldsymbol{H} K K E E--E S F O & \text { SCAFCKPORVTSVLV } & \text { ELDCPGLDPPFRLKK IOKVKOCRCMSVNLS DSDKO }\end{array}$ IPRHVKKE--ESFO SCAFCKPORVTSVLV ELDCPGLDPPFRLKK IOKVKOCRCMSVNLS DSDKO $\begin{array}{llll}P R H V K K E E--E S F Q & \text { SCAFCKPQRVTSVLV } & \text { ELDCPGLDPPFRLKK IQKVKQCRCMSVNLS DSDKQ }\end{array}$ IPRHVKKEE--ESFQ SCAFCKPQRVTSVLV ELECPGLDPPFRLKK IQKVKQCRCMSVNLS DSDKO PR HVKEO--ESFO SCAFCKPORVTSVLV ELECPGLDPPFRLKK IOKVKOCRCMSVNTS DSDKO $\begin{array}{llll}P R \mathbf{H V K E E}--\mathrm{EF} Q & \mathrm{SCAFCKPQRVTSVLV} & \text { ELECPGLDPPFRLKK IQKVKQCRCMSVNLS DSDKO }\end{array}$ 
Figure 4 (on next page)

Schematic representation of the gremlin 2 syntenic region in pigs

Figure 4. Schematic representation of the gremlin 2 syntenic region in pigs. One of the copies (gremlin 2-T1) is located on the 5' side of the regulator of the G-protein signaling 7 gene (RGS7) whereas the second copy (gremlin 2-T2) is located within the RGS7 gene, specifically between exons 13 and 14 . 


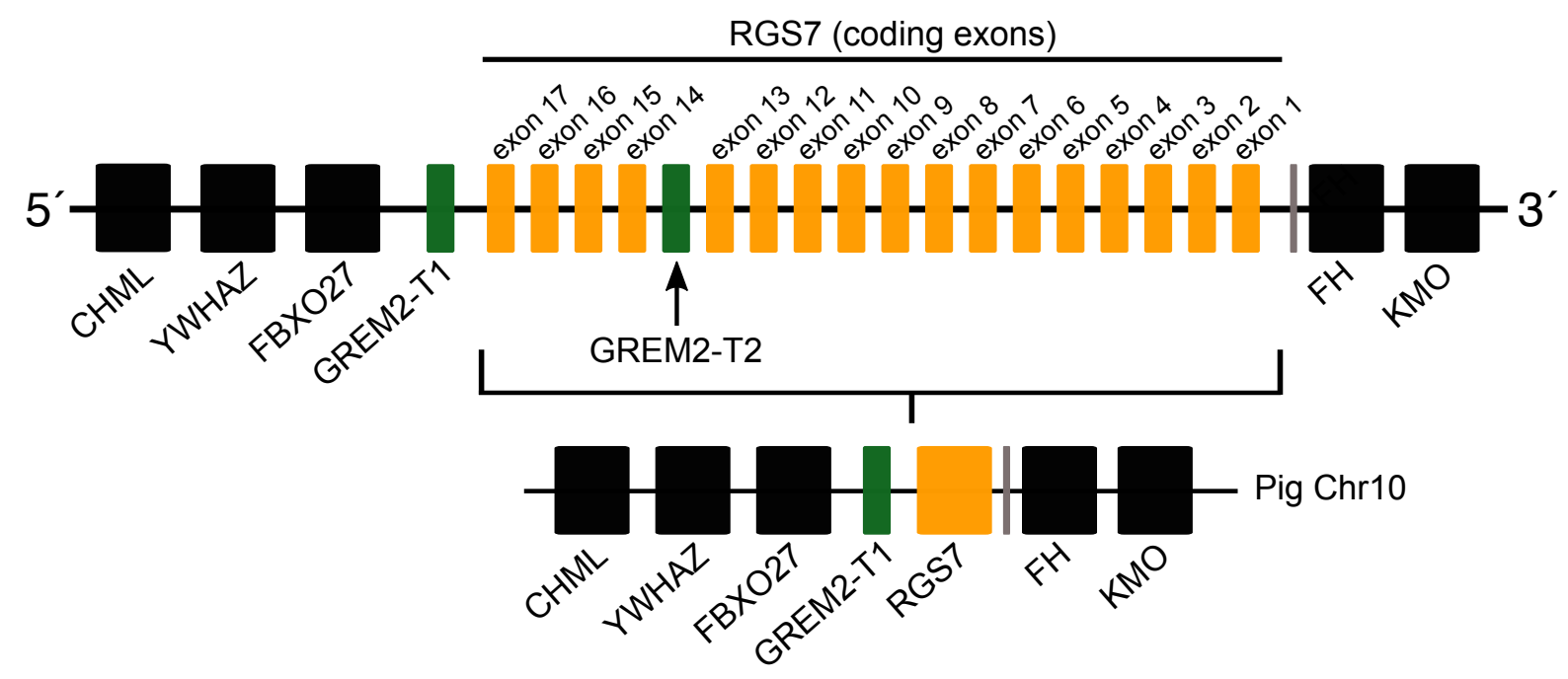




\section{Figure $\mathbf{5}$ (on next page)}

An evolutionary hypothesis regarding the evolution of the gremlin 2 gene in cetartiodactyl mammals

An evolutionary hypothesis regarding the evolution of the gremlin 2 gene in cetartiodactyl mammals. According to this model, the last common ancestor of cetartiodactyls possessed a single copy of the gene. Species belonging to the suborders Tylopoda, the group that includes camels, alpacas, vicuñas and guanacos, and Suiformes, the group that includes pigs and peccaries, were the only groups that retained gremlin 2. According to the phyletic distribution of gremlin 2, we propose that this gene was lost in the common ancestor of ruminants, hippopotamuses, and cetaceans between 56.3 and 63.5 million of years ago as a product of a chromosomal rearrangement. 


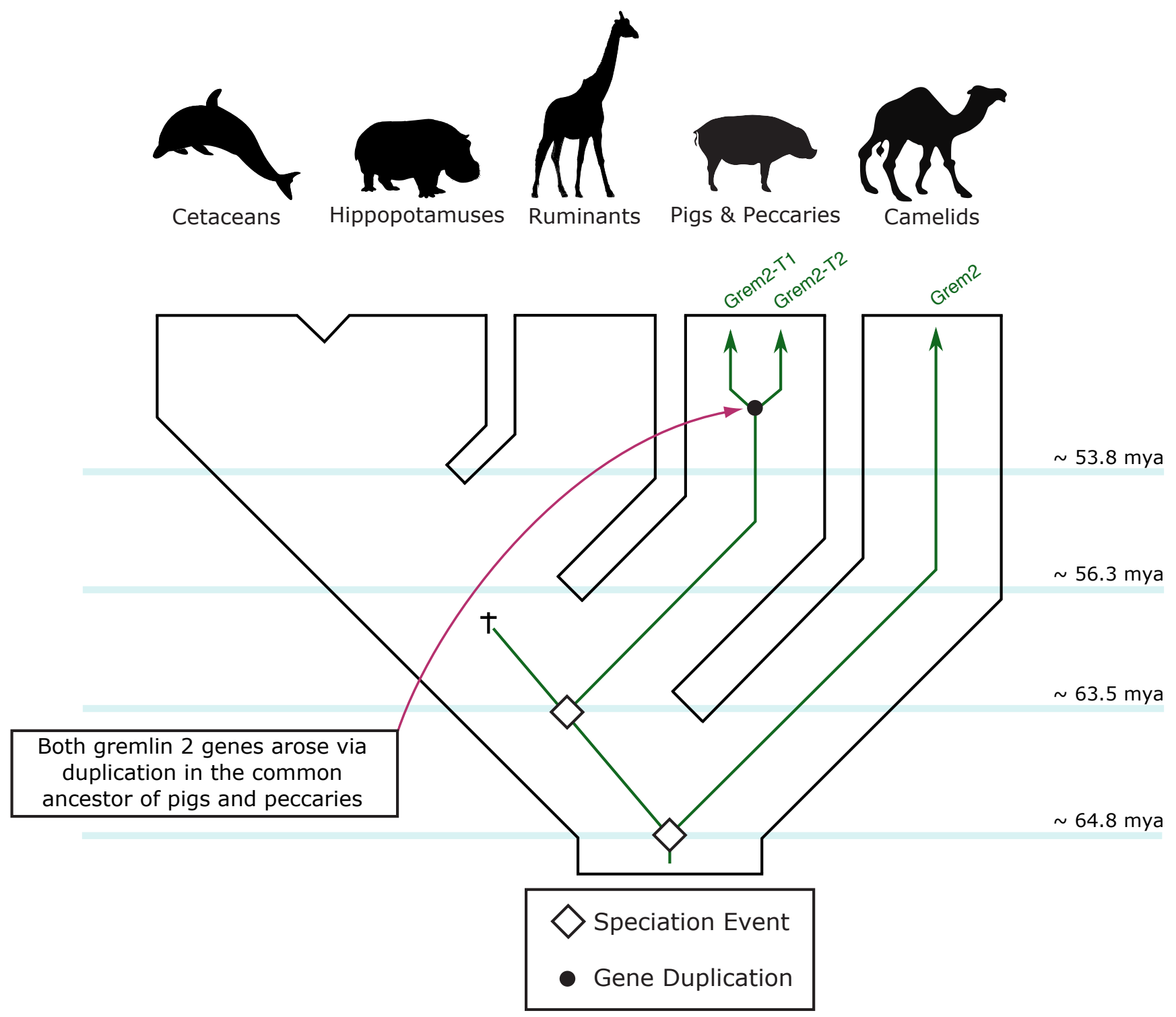




\section{Figure 6 (on next page)}

Schematic representation of the chromosomal regions that harbor genes located upand downstream of gremlin 2

Schematic representation of the chromosomal regions that harbor genes located up- and downstream of gremlin 2. Upper panel: chromosomal region that harbors genes that are upand downstream of gremlin 2 in humans. Middle panel: Chromosomal regions (chrs 16 and 28) that harbor genes that are located up- and downstream of the putative location of gremlin 2 in cow. Lower panel: Chromosomal regions (chrs 25 and 12) that harbor genes that are located up- and downstream of the putative location of gremlin 2 in sheep. 
Peer J

Manuscript to be reviewed

Cow Chr 16/Sheep Chr 12

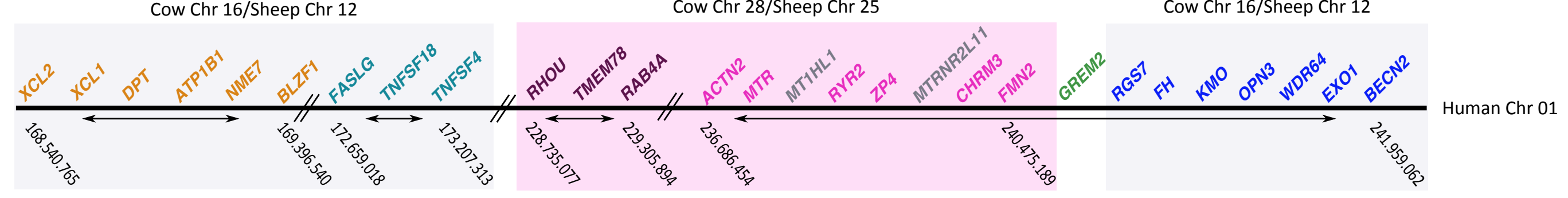

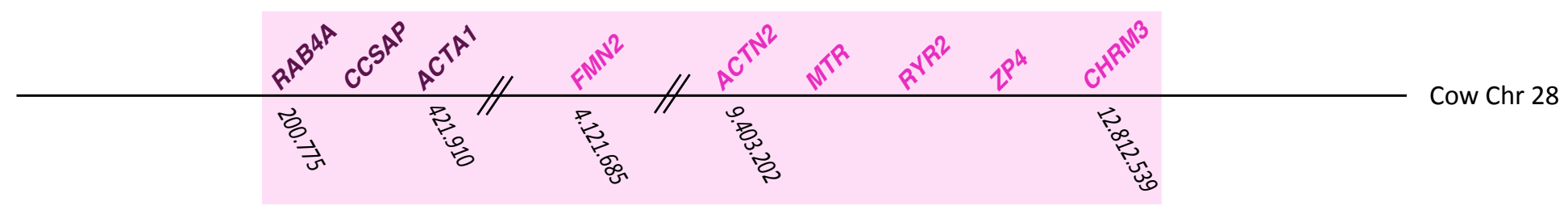
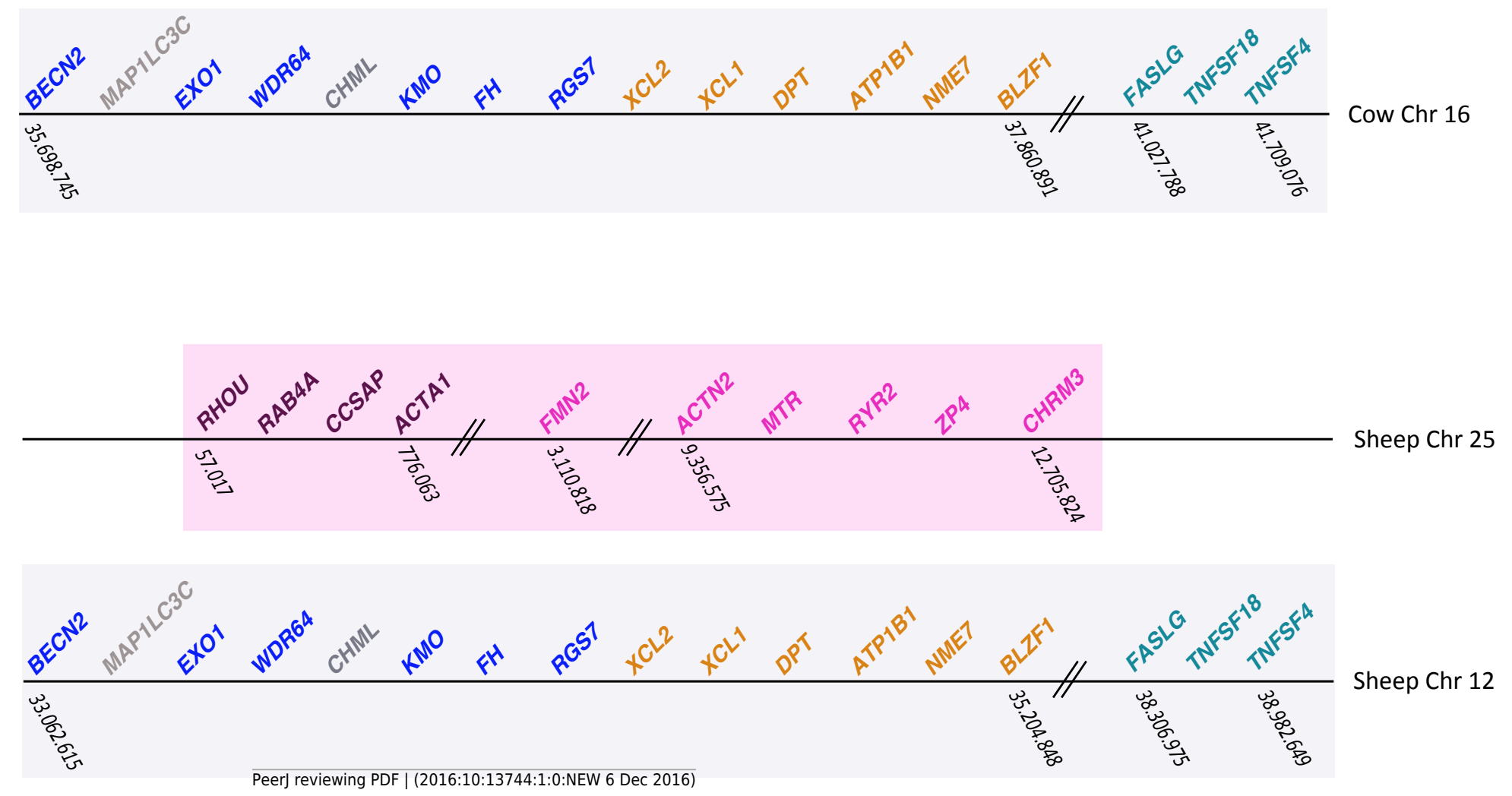